\title{
Higher order commutators of Riesz transforms related to Schrödinger operators
}

Yuanting Wang and Yu Liu*

${ }^{*}$ Correspondence:

liuyu75@pku.org.cn

School of Mathematics and Physics, University of Science and

Technology Beijing, Beijing, 100083, China

\begin{abstract}
Let $L=-\Delta+V$ be a Schrödinger operator on $\mathbb{R}^{d}, d \geq 3$, where the nonnegative potential $V$ belongs to the reverse Hölder class $R H_{a}$ for $q \geq d / 2$. Suppose that $b$ belongs to a new $B M O$ space which is larger than the classical $B M O$ space. We obtain the $L^{p}$ boundedness of higher order commutators $T_{b}^{m}$ defined by $T_{b}^{0}=T$, $T_{b}^{m}=\left[T_{b}^{m-1}, b\right], m=1,2, \ldots$, and $T$ is any of the Riesz transforms or their conjugates associated to the Schrödinger operator $-\Delta+V$. The range of $p$ is related to the index $q$. Moreover, we prove that $T_{b}^{m}$ is bounded from the Hardy space $H_{L}^{1}\left(\mathbb{R}^{d}\right)$ into the space $L_{\text {weak }}^{1}\left(\mathbb{R}^{d}\right)$ when $T$ is the Riesz transform associated to the Schrödinger operator. MSC: 42B20; 35J10
\end{abstract}

Keywords: Schrödinger operators; Riesz transform; BMO; commutators

\section{Introduction}

Let $L=-\Delta+V$ be a Schrödinger operator on $\mathbb{R}^{d}, d \geq 3$, where $V \neq 0$ is a nonnegative potential belonging to a reverse Hölder class $R H_{q}$ for $q>d / 2$. Let $b \in B M O_{\infty}(\rho)$, which is larger than the space $B M O\left(\mathbb{R}^{d}\right)$. In this paper, we consider the Riesz transforms associated with the Schrödinger operator $L$ defined by $\mathcal{R}=\nabla L^{-1 / 2}$ and the higher order commutator

$$
\mathcal{R}_{b}^{m} f(x)=\int_{\mathbb{R}^{d}}(b(x)-b(y))^{m} \mathcal{K}(x, y) f(y) d y,
$$

where $\mathcal{K}(x, y)$ is the kernel of $\mathcal{R}$ and $m=1,2, \ldots$

We also consider its dual transforms associated with the Schrödinger operator $L$ defined by $\tilde{\mathcal{R}}=L^{-1 / 2} \nabla$ and the higher order commutator

$$
\tilde{\mathcal{R}}_{b}^{m} f(x)=\int_{\mathbb{R}^{d}}(b(x)-b(y))^{m} \tilde{\mathcal{K}}(x, y) f(y) d y,
$$

where $\tilde{\mathcal{K}}(x, y)$ is the kernel of $\tilde{\mathcal{R}}$ and $m=1,2, \ldots$

The commutators of singular integral operators have always been one of the hottest problems in harmonic analysis. Recently, some scholars have extended these results to the case of higher order commutators. Please refer to [1-6] and so on. Furthermore, the commutators of singular integral operators related to Schrödinger operators have been brought to many scholars' attention. See, for example, [7-18] and the references therein. Motivated by the references, in this paper we aim to investigate the $L^{p}$ estimates and endpoint estimates for $\mathcal{R}_{b}^{m}$ when $b \in B M O_{\infty}(\rho)$.

○2014 Wang and Liu; licensee Springer. This is an Open Access article distributed under the terms of the Creative Commons Attribution License (http://creativecommons.org/licenses/by/2.0), which permits unrestricted use, distribution, and reproduction in any medium, provided the original work is properly cited. 
Note that a nonnegative locally $L^{q}$ integrable function $V(x)(1<q<\infty)$ on $\mathbb{R}^{d}$ is said to belong to $R H_{q}$ if there exists a constant $C>0$ such that

$$
\left(\frac{1}{|B|} \int_{B} V(x)^{q} d x\right)^{\frac{1}{q}} \leq \frac{C}{|B|} \int_{B} V(x) d x
$$

holds for every ball $B \subset \mathbb{R}^{d}$. It is known that $V \in R H_{q}$ implies $V \in R H_{q+\varepsilon}$ for some $\varepsilon>0$. Therefore, under the assumption $V \in R H_{q_{0}}$, we may conclude $q_{0}>d / 2$.

We introduce the auxiliary function $\rho$ defined as, for $x \in \mathbb{R}^{d}$,

$$
\rho(x)=\frac{1}{m(x, V)}=\sup _{r>0}\left\{r: \frac{1}{r^{d-2}} \int_{B(x, r)} V(y) d y \leq 1\right\} .
$$

The class $B M O_{\theta}(\rho)$ of locally integrable functions $b$ is defined as follows:

$$
\frac{1}{|B(x, r)|} \int_{B(x, r)}\left|b(y)-b_{B}\right| d y \leq C\left(1+\frac{r}{\rho(x)}\right)^{\theta},
$$

for all $x \in \mathbb{R}^{d}$ and $r>0$, where $\theta>0$ and $b_{B}=\frac{1}{|B|} \int_{B} b$. A norm for $b \in B M O_{\theta}(\rho)$ denoted by $[b]_{\theta}$ is given by the infimum of the constants satisfying (2) after identifying functions that differ upon a constant. Denote that $B M O_{\infty}(\rho)=\bigcup_{\theta>0} B M O_{\theta}(\rho)$. It is easy to see that $B M O\left(\mathbb{R}^{d}\right) \subset B M O_{\theta}(\rho) \subset B M O_{\theta}^{\prime}(\rho)$ for $0<\theta<\theta^{\prime}$. Bongioanni et al. [8] gave some examples to clarify that the space $B M O\left(\mathbb{R}^{d}\right)$ is a subspace of $B M O_{\infty}(\rho)$.

Because $V \geq 0$ and $V \in L_{\text {loc }}^{\frac{d}{2}}\left(\mathbb{R}^{d}\right)$, the Schrödinger operator $L$ generates a $\left(C_{0}\right)$ contraction semigroup $\left\{T_{s}^{L}: s>0\right\}=\left\{e^{-s L}: s>0\right\}$. The maximal function associated with $\left\{T_{s}^{L}: s>0\right\}$ is defined by $M^{L} f(x)=\sup _{s>0}\left|T_{s}^{L} f(x)\right|$. The Hardy space $H_{L}^{1}\left(\mathbb{R}^{d}\right)$ associated with the Schrödinger operator $L$ is defined as follows in terms of the maximal function mentioned.

Definition 1 A function $f \in L^{1}\left(\mathbb{R}^{d}\right)$ is said to be in $H_{L}^{1}\left(\mathbb{R}^{d}\right)$ if the maximal function $M^{L} f$ belongs to $L^{1}\left(\mathbb{R}^{d}\right)$. The norm of such a function is defined by

$$
\|f\|_{H_{L}^{1}}=\left\|M^{L} f\right\|_{L^{1}} .
$$

Definition 2 Let $1<q \leq \infty$. A measurable function $a$ is called a $(1, q)_{\rho}$-atom associated to the ball $B(x, r)$ if $r<\rho(x)$ and the following conditions hold:

(1) $\operatorname{supp} a \subset B(x, r)$;

(2) $\|a\|_{L^{q}\left(\mathbb{R}^{d}\right)} \leq|B(x, r)|^{1 / q-1}$;

(3) if $r<\rho(x) / 4, \int_{\mathbb{R}^{d}} a(x) d x=0$.

The space $H_{L}^{1}\left(\mathbb{R}^{n}\right)$ admits the following atomic decomposition (cf. [19]).

Proposition 1 Let $f \in L^{1}\left(\mathbb{R}^{d}\right)$. Then $f \in H_{L}^{1}\left(\mathbb{R}^{d}\right)$ if and only iff can be written as $f=\sum_{j} \lambda_{j} a_{j}$, where $a_{j}$ are $(1, q)_{\rho}$-atoms and $\sum_{j}\left|\lambda_{j}\right|<\infty$. Moreover,

$$
\|f\|_{H_{L}^{1}} \sim \inf \left\{\sum_{j}\left|\lambda_{j}\right|\right\}
$$

where the infimum is taken over all atomic decompositions off into $H_{L}^{1}$-atoms. 
Before stating the main theorems, we introduce the definition of the reverse Hölder index of $V$ as $q_{0}=\sup \left\{q: V \in R H_{q}\right\}$ (cf. [8]). In what follows, we state our main results in this paper.

Theorem 1 Let $V \in R H_{d / 2}, b \in B M O_{\infty}(\rho)$ and $p_{0}$ such that $1 / p_{0}=\left(1 / q_{0}-1 / d\right)^{+}$, where $q_{0}$ is the reverse Hölder index of $V$. If $p_{0}^{\prime}<p<\infty$, then

$$
\left\|\tilde{\mathcal{R}}_{b}^{m} f\right\|_{L^{p}} \lesssim\left(\sum_{\alpha=1}^{m}[b]_{\theta}^{\alpha}\right)\|f\|_{L^{p}}
$$

where $\left(1 / p_{0}\right)+\left(1 / p_{0}^{\prime}\right)=1$.

By duality, we immediately have the following theorem.

Theorem 2 Let $V \in R H_{d / 2}, b \in B M O_{\infty}(\rho)$ and $p_{0}$ such that $1 / p_{0}=\left(1 / q_{0}-1 / d\right)^{+}$, where $q_{0}$ is the reverse Hölder index of $V$. If $1<p<p_{0}$, then

$$
\left\|\mathcal{R}_{b}^{m} f\right\|_{L^{p}} \lesssim\left(\sum_{\alpha=1}^{m}[b]_{\theta}^{\alpha}\right)\|f\|_{L^{p}},
$$

where $\left(1 / p_{0}\right)+\left(1 / p_{0}^{\prime}\right)=1$.

Theorem 3 Suppose that $V \in R H_{q}$ for some $q \geq \frac{d}{2}$. Let $b \in B M O_{\infty}(\rho)$. Then, for any $\lambda>0$, we have

$$
\left|\left\{x \in \mathbb{R}^{d}:\left|\mathcal{R}_{b}^{m}(f)(x)\right|>\lambda\right\}\right| \lesssim \frac{\left(\sum_{\alpha=1}^{m}[b]_{\theta}^{\alpha}\right)}{\lambda}\|f\|_{H_{L}^{1}\left(\mathbb{R}^{d}\right)}, \quad \forall f \in H_{L}^{1}\left(\mathbb{R}^{d}\right) .
$$

Namely, the commutator $\mathcal{R}_{b}^{m}$ is bounded from $H_{L}^{1}\left(\mathbb{R}^{d}\right)$ into $L_{\text {weak }}^{1}\left(\mathbb{R}^{d}\right)$.

The proofs of Theorems 1 and 2 can be given by iterating $m$ times starting from Lemmas 12 and 13. Please refer to Section 3 for details.

Throughout this paper, unless otherwise indicated, we always assume that $0 \neq V \in R H_{q}$ for some $q>d / 2$. We will use $C$ to denote a positive constant, which is not necessarily the same at each occurrence. By $A \sim B$ and $A \lesssim B$, we mean that there exist some positive constants $C, C^{\prime}$ such that $1 / C \leq A / B \leq C$ and $A \leq C^{\prime} B$, respectively.

\section{Some lemmas}

In this section, we collect some known results about the auxiliary function $\rho(x)$ and some necessary estimates for the kernel of the Riesz transform in the paper (cf. [20] or [7]). In the end, we recall some propositions and lemmas for the $B M O$ spaces $B M O_{\theta}(\rho)$ in [8].

Lemma $1 V \in R H_{q}$ for some $q>d / 2$ implies that $V$ satisfies the doubling condition; that is, there exists a constant $C>0$ such that

$$
\int_{B(x, 2 r)} V(y) d y \leq C \int_{B(x, r)} V(y) d y .
$$


Especially, there exist constants $\mu \geq 1$ and $C$ such that

$$
\int_{B(x, t r)} V(y) d y \leq C t^{d \mu} \int_{B(x, r)} V(y) d y
$$

holds for every ball $B(x, r)$ and $t>1$.

Lemma 2 Let $V \in R H_{d / 2}$. For the auxiliary function $\rho$, there exist $C>0$ and $l_{0} \geq 1$ such that

$$
C^{-1} \rho(x)\left(1+\frac{|x-y|}{\rho(x)}\right)^{-l_{0}} \leq \rho(y) \leq C \rho(x)\left(1+\frac{|x-y|}{\rho(x)}\right)^{\frac{l_{0}}{l_{0}+1}}
$$

for all $x, y \in \mathbb{R}^{d}$.

In particular, $\rho(y) \sim \rho(x)$ if $|x-y|<C \rho(x)$.

Lemma 3 If $V \in R H_{d / 2}$, then there exists $C>0$ such that

$$
\int_{B(x, R)} \frac{V(y) d y}{|x-y|^{d-2}} \leq \frac{C}{R^{d-2}} \int_{B(x, R)} V(y) d y .
$$

Moreover, if $V \in R H_{d}$, then there exists $C>0$ such that

$$
\int_{B(x, R)} \frac{V(y) d y}{|x-y|^{d-1}} \leq \frac{C}{R^{d-1}} \int_{B(x, R)} V(y) d y .
$$

Lemma 4 For $0<r<R<\infty$,

$$
\frac{1}{r^{d-2}} \int_{B(x, r)} V(y) d y \leq C\left(\frac{r}{R}\right)^{2-d / q} \frac{1}{R^{d-2}} \int_{B(x, R)} V(y) d y .
$$

It is easy to see that

$$
\frac{1}{r^{d-2}} \int_{B(x, r)} V(y) d y \sim 1 \quad \text { if } r \sim \rho(x) .
$$

Lemma 5 There exist constants $C>0$ and $l_{0}^{\prime}>0$ such that

$$
\frac{1}{R^{d-2}} \int_{B(x, R)} V(y) d y \leq C\left(1+\frac{R}{\rho(x)}\right)^{l_{0}^{\prime}} .
$$

Lemma 6 Let $\theta>0$ and $1 \leq s<\infty$. If $b \in B M O_{\theta}(\rho)$, then

$$
\left(\frac{1}{|B|} \int_{B}\left|b-b_{B}\right|^{s}\right)^{1 / s} \lesssim[b]_{\theta}\left(1+\frac{r}{\rho(x)}\right)^{\theta^{\prime}}
$$

for all $B=B(x, r)$, with $x \in \mathbb{R}^{d}$ and $r>0$, where $\theta^{\prime}=\left(l_{0}+1\right) \theta$ and $l_{0}$ is the constant appearing in (4). 
Lemma 7 Let $b \in B M O_{\theta}(\rho), B=B\left(x_{0}, r\right)$ and $s \geq 1$. Then

$$
\left(\frac{1}{\left|2^{k} B\right|} \int_{2^{k} B}\left|b-b_{B}\right|^{s}\right)^{1 / s} \lesssim[b]_{\theta} k\left(1+\frac{2^{k} r}{\rho\left(x_{0}\right)}\right)^{\theta^{\prime}}
$$

for all $k \in \mathrm{N}$ with $\theta^{\prime}=\left(l_{0}+1\right) \theta$.

Lemma 8 If $V \in R H_{s}$ for $s>\frac{d}{2}$, then we have the following:

(i) for every $N$, there exists a constant $C_{N}>0$ such that

$$
|\mathcal{K}(x, y)| \leq \frac{C_{N}}{\left(1+|x-y| \rho(x)^{-1}\right)^{N}}\left(\frac{1}{|x-y|^{d-1}} \int_{B(y,|x-y|)} \frac{V(z) d z}{|z-y|^{d-1}}+\frac{1}{|x-y|^{d}}\right)
$$

and

(ii) for every $N$, there exists a constant $C_{N}>0$ such that

$$
\begin{aligned}
& |\mathcal{K}(x, y+h)-\mathcal{K}(x, y)| \\
& \quad \leq \frac{C_{N}}{\left(1+|x-y| \rho(x)^{-1}\right)^{N}} \frac{|h|^{\delta}}{|x-y|^{d-1+\delta}}\left(\int_{B(y,|x-y|)} \frac{V(z) d z}{|z-y|^{d-1}}+\frac{1}{|x-y|}\right)
\end{aligned}
$$

for some $\delta>0$, whenever $|h|<\frac{1}{16}|x-y|$.

Lemma 9 If $V \in R H_{d / 2}$, then we have the following:

(i) For every $N$, there exists a constant $C_{N}>0$ such that

$$
|\tilde{\mathcal{K}}(x, z)| \leq \frac{C_{N}\left(1+\frac{|x-z|}{\rho(x)}\right)^{-N}}{|x-z|^{d-1}}\left(\int_{B(z,|x-z| / 4)} \frac{V(u)}{|u-z|^{d-1}} d u+\frac{1}{|x-z|}\right)
$$

Moreover, the last inequality also holds with $\rho(x)$ replaced by $\rho(z)$.

(ii) For every $N$, there exists a constant $C_{N}>0$ such that

$$
|\tilde{\mathcal{K}}(x, y+h)-\tilde{\mathcal{K}}(x, y)| \leq \frac{C_{N}|h|^{\delta}\left(1+\frac{|x-y|}{\rho(x)}\right)^{-N}}{|x-y|^{d-1+\delta}}\left(\int_{B(y,|x-y| / 4)} \frac{V(u)}{|u-y|^{d-1}} d u+\frac{1}{|x-y|}\right),
$$

whenever $|h|<\frac{1}{16}|x-y|$. Moreover, the last inequality also holds with $\rho(x)$ replaced by $\rho(y)$.

(iii) If $K^{*}$ denotes the $\mathbb{R}^{d}$ vector-valued kernel of the adjoint of the classical Riesz operator, then for some $0<\delta<2-\frac{d}{s}$,

$$
\left|\tilde{\mathcal{K}}(x, z)-K^{*}(x, z)\right| \leq \frac{C}{|x-z|^{d-1}}\left(\int_{B(z,|x-z| / 4)} \frac{V(u)}{|u-z|^{d-1}} d u+\frac{1}{|x-z|}\left(\frac{|x-z|}{\rho(x)}\right)^{\delta}\right),
$$

whenever $|x-z|<\rho(x)$.

(iv) When $s>d$, the term involving $V$ can be dropped from inequalities (10), (11) and (12).

Proposition 2 (cf. Theorem 0.5 in [20]) Suppose that $V \in R H_{s}$ for some $s>d / 2$, then

(i) $\tilde{\mathcal{R}}$ is bounded on $L^{p}\left(\mathbb{R}^{d}\right)$ for $p_{0}^{\prime}<p<\infty$;

(ii) $\mathcal{R}$ is bounded on $L^{p}\left(\mathbb{R}^{d}\right)$ for $1<p<p_{0}$, where $1 / p_{0}=(1 / s-1 / d)^{+}$. 
Proposition 3 (cf. Theorem 1 in [8]) Suppose that $V \in R H_{s}$ for some $s>d / 2$ and $b \in$ $B M O_{\infty}(\rho)$, then

(i) $\tilde{\mathcal{R}}_{b}$ is bounded on $L^{p}\left(\mathbb{R}^{d}\right)$ for $p_{0}^{\prime}<p<\infty$;

(ii) $\mathcal{R}_{b}$ is bounded on $L^{p}\left(\mathbb{R}^{d}\right)$ for $1<p<p_{0}$,

where $1 / p_{0}=(1 / s-1 / d)^{+}$.

A ball $B(x, \rho(x))$ is called critical. In [19], Dziubański and Zienkiewicz gave the following covering lemma on $\mathbb{R}^{d}$.

Lemma 10 There exists a sequence of points $\left\{x_{k}\right\}_{k=1}^{\infty}$ in $\mathbb{R}^{d}$ such that the family of critical balls $Q_{k}=B\left(x_{k}, \rho\left(x_{k}\right)\right), k \geq 1$, satisfies the following:

(i) $\bigcup_{k} Q_{k}=\mathbb{R}^{d}$.

(ii) There exists $N=N(\rho)$ such that for every $k \in N$,

$\operatorname{card}\left\{j: 4 Q_{j} \cap 4 Q_{k} \neq \emptyset\right\} \leq N$.

Given that $\alpha>0$, we define the following maximal functions for $g \in L_{\mathrm{loc}}^{1}\left(\mathbb{R}^{d}\right)$ and $x \in \mathbb{R}^{d}$ :

$$
\begin{aligned}
& M_{\rho, \alpha} g(x)=\sup _{x \in B \in \mathcal{B}_{\rho, \alpha}} \frac{1}{|B|} \int_{B}|g|, \\
& M_{\rho, \alpha}^{\#} g(x)=\sup _{x \in B \in \mathcal{B}_{\rho, \alpha}} \frac{1}{|B|} \int_{B}\left|g-g_{B}\right|,
\end{aligned}
$$

where $\mathcal{B}_{\rho, \alpha}=\left\{B(y, r): y \in \mathbb{R}^{d}, r \leq \alpha \rho(y)\right\}$.

Also, given a ball $Q \subset \mathbb{R}^{d}$, for $g \in L_{\text {loc }}^{1}\left(\mathbb{R}^{d}\right)$ and $y \in Q$, we define

$$
\begin{aligned}
& M_{Q} g(x)=\sup _{x \in B \in \mathcal{F}(Q)} \frac{1}{|B \cap Q|} \int_{B \cap Q}|g|, \\
& M_{Q}^{\#} g(x)=\sup _{x \in B \in \mathcal{F}(Q)} \frac{1}{|B \cap Q|} \int_{B \cap Q}\left|g-g_{B \cap Q}\right|,
\end{aligned}
$$

where $\mathcal{F}(Q)=\{B(y, r): y \in Q, r>0\}$.

Lemma 11 (Fefferman-Stein type inequality, $c f$. Lemma 2 in [8]) For $1<\rho<\infty$, there exist $\beta$ and $\gamma$ such that if $\left\{Q_{k}\right\}_{k=1}^{\infty}$ is a sequence of balls as in Lemma 10, then

$$
\int_{\mathbb{R}^{d}}\left|M_{\rho, \beta}(g)\right|^{p} \lesssim \int_{\mathbb{R}^{d}}\left|M_{\rho, \gamma}^{\#}(g)\right|^{p}+\sum_{k}\left|Q_{k}\right|\left(\frac{1}{\left|Q_{k}\right|} \int_{2 Q_{k}}|g|\right)^{p}
$$

for all $g \in L_{\mathrm{loc}}^{1}\left(\mathbb{R}^{d}\right)$.

\section{Proofs of the main results}

Firstly, in order to prove the main theorems, we need the following lemmas. As usual, for $f \in L_{\text {loc }}^{1}\left(\mathbb{R}^{d}\right)$, we denote by $M_{p}$ the $p$-maximal function which is defined as

$$
M_{p} f(x)=\sup _{r>0}\left(\frac{1}{|B(x, r)|} \int_{B(x, r)}|f(y)|^{p} d y\right)^{1 / p} .
$$


Lemma 12 Let $V \in R H_{s}$ for some $s \geq d / 2,1 / p_{0}=(1 / s-1 / d)^{+}$, and $b \in B M O_{\theta}(\rho)$. Then, for any $p>p_{0}^{\prime}$, there exists a constant $C_{m}>0$ such that

$$
\frac{1}{|Q|} \int_{Q}\left|\tilde{\mathcal{R}}_{b}^{m} f\right| \leq C_{m}\left(\sum_{\alpha=1}^{m}[b]_{\theta}^{\alpha}\right)\left[\inf _{y \in Q} M_{p} f(y)+\inf _{y \in Q} M_{p}\left(\tilde{\mathcal{R}}_{b}^{\alpha} f\right)(y)\right]
$$

for all $f \in L_{\mathrm{loc}}^{p}\left(\mathbb{R}^{d}\right)$ and every ball $Q=B\left(x_{0}, \rho\left(x_{0}\right)\right)$.

Proof We only consider the case of $\frac{d}{2}<s<d$ because the proof of the case of $s>d$ can be easily deduced from that of the case of $\frac{d}{2}<s<d$.

Following (4.5) in [21], we expand $b(x)-b(y)=(b(x)-\lambda)-(b(y)-\lambda)$, where $\lambda$ is an arbitrary constant, as follows:

$$
\begin{aligned}
\tilde{\mathcal{R}}_{b}^{m} f(x)= & \int_{\mathbb{R}^{d}}(b(x)-b(y))^{m} \tilde{\mathcal{K}}(x-y) f(y) d y \\
= & \sum_{j=0}^{m} C_{j, m}(b(x)-\lambda)^{j} \int_{\mathbb{R}^{d}}(b(y)-\lambda)^{m-j} \tilde{\mathcal{K}}(x-y) f(y) d y \\
= & \sum_{j=1}^{m} C_{j, m}(b(x)-\lambda)^{j} \int_{\mathbb{R}^{d}}(b(y)-\lambda)^{m-j} \tilde{\mathcal{K}}(x-y) f(y) d y \\
& +\tilde{\mathcal{R}}\left((b-\lambda)^{m} f\right)(x) \\
= & \sum_{j=1}^{m} C_{j, m}(b(x)-\lambda)^{j} \int_{\mathbb{R}^{d}}(b(y)-b(x)+b(x)-\lambda)^{m-j} \tilde{\mathcal{K}}(x-y) f(y) d y \\
& +\tilde{\mathcal{R}}\left((b-\lambda)^{m} f\right)(x) \\
= & \sum_{j=1}^{m} \sum_{h=0}^{m-j} C_{j, m, h}(b(x)-\lambda)^{j+h} \int_{\mathbb{R}^{d}}(b(x)-b(y))^{m-j-h} \tilde{\mathcal{K}}(x-y) f(y) d y \\
& +\tilde{\mathcal{R}}\left((b-\lambda)^{m} f\right)(x) \\
= & \sum_{\alpha=0}^{m-1} C_{\alpha, m}(b(x)-\lambda)^{m-\alpha} \tilde{\mathcal{R}}_{b}^{\alpha} f(x)+\tilde{\mathcal{R}}\left((b-\lambda)^{m} f\right)(x) \\
= & I_{1}+I_{2} .
\end{aligned}
$$

Let $f \in L^{p}\left(\mathbb{R}^{d}\right)$ and $Q=B\left(x_{0}, \rho\left(x_{0}\right)\right)$ with $\lambda=b_{2 B}$, then we have to deal with the average on $Q$ of each term.

Firstly, by the Hölder inequality with $p>p_{0}^{\prime}$ and Lemma 7,

$$
\begin{aligned}
\frac{1}{|Q|} \int_{Q}\left|I_{1}\right| & \lesssim \sum_{\alpha=0}^{m-1} \frac{1}{|Q|} \int_{Q}\left|\left(b(y)-b_{2 B}\right)^{m-\alpha} \tilde{\mathcal{R}}_{b}^{\alpha} f(y)\right| d y \\
& \lesssim \sum_{\alpha=0}^{m-1}\left(\frac{1}{|Q|} \int_{Q}\left|\left(b(y)-b_{2 B}\right)^{(m-\alpha) p^{\prime}}\right| d y\right)^{1 / p^{\prime}}\left(\frac{1}{|Q|} \int_{Q}\left|\tilde{\mathcal{R}}_{b}^{\alpha} f\right|^{p}\right)^{1 / p} \\
& \lesssim\left(\sum_{\alpha=0}^{m-1}\left([b]_{\theta}\right)^{m-\alpha}\right) \inf _{y \in Q} M_{p}\left(\tilde{\mathcal{R}}_{b}^{\alpha} f\right)(y) .
\end{aligned}
$$


As for $I_{2}$, we split $f=f_{1}+f_{2}$. Choosing $p_{0}^{\prime}<\tilde{p}<p$ and denoting $\frac{1}{\tilde{p}^{\prime}}+\frac{1}{\tilde{p}}=1$ and $v=\frac{\tilde{p} p}{p-\tilde{p}}$, using the boundedness of $\tilde{\mathcal{R}}$ on $L^{\tilde{p}}\left(\mathbb{R}^{d}\right)$ and the Hölder inequality, we obtain

$$
\begin{aligned}
\frac{1}{|Q|} \int_{Q}\left|\tilde{\mathcal{R}}\left(\left(b(x)-b_{2 B}\right)^{m} f_{1}\right)\right| & \lesssim\left(\frac{1}{|Q|} \int_{Q}\left|\tilde{\mathcal{R}}\left(\left(b(x)-b_{2 B}\right)^{m} f_{1}\right)\right|^{\tilde{p}}\right)^{1 / \tilde{p}} \\
& \lesssim\left(\frac{1}{|Q|} \int_{2 Q}\left|\left(b(x)-b_{2 B}\right)^{m} f\right|^{\tilde{p}}\right)^{1 / \tilde{p}} \\
& \lesssim\left(\frac{1}{|Q|} \int_{2 Q}|f|^{p}\right)^{1 / p}\left(\frac{1}{|Q|} \int_{2 Q}\left|\left(b(x)-b_{2 B}\right)\right|^{m v}\right)^{1 / v} \\
& \lesssim[b]_{\theta}^{m} \inf _{y \in Q} M_{p} f(y),
\end{aligned}
$$

where in the last inequality we have used Lemma 6 for the remaining term. We firstly note the fact that $\rho(x) \sim \rho\left(x_{0}\right)$ and $|x-z| \sim\left|x_{0}-z\right|$. Then we have to deal with

$$
\left|\tilde{\mathcal{R}}\left(\left(b-b_{2 B}\right)^{m} f_{2}\right)\right|=\left|\int_{\left|x_{0}-z\right|>2 \rho\left(x_{0}\right)} \tilde{\mathcal{K}}\left(x_{0}-z\right)\left(b(z)-b_{2 B}\right)^{m} f(z) d z\right| \lesssim \tilde{I}_{1}(x)+\tilde{I}_{2}(x),
$$

where

$$
\begin{aligned}
& \tilde{I}_{1}(x)=\int_{\left|x_{0}-z\right|>2 \rho\left(x_{0}\right)} \frac{\left|b(z)-b_{2 B}\right|^{m}|f(z)|}{\left(1+\frac{\left|x_{0}-z\right|}{\rho\left(x_{0}\right)}\right)^{N}|x-z|^{d}} d z, \\
& \tilde{I}_{2}(x)=\int_{\left|x_{0}-z\right|>2 \rho\left(x_{0}\right)} \frac{\left|b(z)-b_{2 B}\right|^{m}|f(z)|}{\left(1+\frac{\left|x_{0}-z\right|}{\rho\left(x_{0}\right)}\right)^{N}|x-z|^{d-1}} \int_{B\left(z,\left|x_{0}-z\right| / 4\right)} \frac{|V(u)|}{|u-z|^{d-1}} d u d z .
\end{aligned}
$$

For $\tilde{I}_{1}(x)$, we have

$$
\begin{aligned}
\tilde{I}_{1}(x) & \lesssim \sum_{j=1}^{\infty}\left(1+2^{j}\right)^{-N}\left(2^{j-1} \rho\left(x_{0}\right)\right)^{-d} \int_{2^{j} \rho\left(x_{0}\right)<\left|x_{0}-z\right| \leq 2^{j+1} \rho\left(x_{0}\right)}\left|b(z)-b_{2 B}\right|^{m}|f(z)| d z \\
& \lesssim\left([b]_{\theta}\right)^{m} \inf _{y \in Q} M_{S} f(y) \sum_{j=1}^{\infty} j^{m} 2^{j\left(-N+m \theta^{\prime}\right)} \\
& \lesssim\left([b]_{\theta}\right)^{m} \inf _{y \in Q} M_{S} f(y),
\end{aligned}
$$

where we have used the following inequality:

$$
\begin{aligned}
& \left\|f\left(b-b_{2 B}\right)^{m} \chi_{B\left(x_{0}, 2^{j} \rho\left(x_{0}\right)\right)}\right\|_{1} \leq\left\|f \chi_{B\left(x_{0}, 2^{j} \rho\left(x_{0}\right)\right)}\right\|_{p}\left\|\left(b-b_{2 B}\right)^{m} \chi_{B\left(x_{0}, 2^{j} \rho\left(x_{0}\right)\right)}\right\|_{p^{\prime}} \\
& \lesssim\left(2^{j} \rho\left(x_{0}\right)\right)^{d} \inf _{y \in Q} M_{p} f(y)\left(j 2^{j \theta^{\prime}}[b]_{\theta}\right)^{m}
\end{aligned}
$$

by using Lemma 7, and we choose $N$ large enough. As for $\tilde{I}_{2}(x)$,

$$
\begin{aligned}
\tilde{I}_{2}(x) \lesssim & \sum_{j=1}^{\infty}\left(1+2^{j}\right)^{-N} 2^{j} \rho\left(x_{0}\right) \frac{1}{\left|B\left(x_{0}, 2^{j} \rho\left(x_{0}\right)\right)\right|} \\
& \times \int_{2^{j} \rho\left(x_{0}\right)<\left|x_{0}-z\right| \leq 2^{j+1} \rho\left(x_{0}\right)}\left|b(z)-b_{2 B}\right|^{m}\left|f_{2}(z)\right| \int_{B\left(z, 2^{j+3} \rho\left(x_{0}\right)\right)} \frac{|V(u)|}{|u-z|^{d-1}} d u d z
\end{aligned}
$$




$$
\begin{aligned}
\lesssim & \sum_{j=1}^{\infty}\left(1+2^{j}\right)^{-N} 2^{j} \rho\left(x_{0}\right) \frac{1}{\left|B\left(x_{0}, 2^{j} \rho\left(x_{0}\right)\right)\right|} \\
& \times \int_{2^{j} \rho\left(x_{0}\right)<\left|x_{0}-z\right| \leq 2^{j+1} \rho\left(x_{0}\right)}\left|b(z)-b_{2 B}\right|^{m}\left|f_{2}(z)\right| \mathcal{F}_{1}\left(|V| \chi_{\left.B\left(z, 2^{j+3} \rho\left(x_{0}\right)\right)\right)}\right) d z .
\end{aligned}
$$

Using the Hölder inequality and the boundedness of the fractional integral $\mathcal{F}_{1}$ with $1 / p^{\prime}=$ $1 / s-1 / d$, we obtain

$$
\begin{aligned}
& \int_{2^{j} \rho\left(x_{0}\right)<\left|x_{0}-z\right| \leq 2^{j+1} \rho\left(x_{0}\right)}\left|b(z)-b_{2 B}\right|^{m}\left|f_{2}(z)\right| \mathcal{F}_{1}\left(|V| \chi_{B\left(z, j^{j+3} \rho\left(x_{0}\right)\right)}\right) d z \\
& \quad \lesssim\left\|f_{2}(z)\left(b-b_{2 B}\right)^{m} \chi_{B\left(z, 2^{j+3} \rho\left(x_{0}\right)\right)}\right\|\left\|_{p}\right\| \mathcal{F}_{1}\left(|V| \chi_{\left.B\left(z, 2^{j+3} \rho\left(x_{0}\right)\right)\right)} \|_{p^{\prime}}\right. \\
& \quad \lesssim\left\|f_{2}(z)\left(b-b_{2 B}\right)^{m} \chi_{B\left(z, j^{j+3} \rho\left(x_{0}\right)\right)}\right\|_{p}\left\||V| \chi_{\left.B\left(z, 2^{j+3} \rho\left(x_{0}\right)\right)\right)}\right\|_{s^{*}}
\end{aligned}
$$

Since $V \in R H_{s}$,

$$
\begin{aligned}
\left\||V| \chi_{B\left(z, 2^{j+3} \rho\left(x_{0}\right)\right)}\right\|_{s} & \lesssim\left(2^{j} \rho\left(x_{0}\right)\right)^{-d / s^{\prime}} \int_{B\left(z, 2^{j} \rho\left(x_{0}\right)\right)}|V(z)| d z \\
& \lesssim\left(2^{j} \rho\left(x_{0}\right)\right)^{d-2-d / s^{\prime}} \frac{1}{\left(2^{j} \rho\left(x_{0}\right)\right)^{d-2}} \int_{B\left(z, 2^{j} \rho\left(x_{0}\right)\right)}|V(z)| d z \\
& \lesssim\left(2^{j} \rho\left(x_{0}\right)\right)^{d-2-d / s^{\prime}}\left(2^{j}\right)^{2-d / s} .
\end{aligned}
$$

And when $v=\frac{\tilde{p} p}{p-\tilde{p}}$ and $1 / p^{\prime}=1 / s-1 / d$, we also have

$$
\begin{aligned}
\left\|f_{2}(z)\left(b-b_{2 B}\right)^{m} \chi_{B\left(z, 2^{j+3} \rho\left(x_{0}\right)\right)}\right\|_{p} & \leq\left\|f \chi_{B\left(x_{0}, 2 j \rho\left(x_{0}\right)\right)}\right\|_{\tilde{p}}\left\|\left(b-b_{2 B}\right)^{m} \chi_{B\left(x_{0}, 2^{j} \rho\left(x_{0}\right)\right)}\right\|_{v} \\
& \lesssim\left(2^{j} \rho\left(x_{0}\right)\right)^{d / p} \inf _{y \in Q} M_{p} f(y)\left(j 2^{j \theta^{\prime}}[b]_{\theta}\right)^{m} \\
& \lesssim\left(j 2^{j \theta^{\prime}}[b]_{\theta}\right)^{m}\left(2^{j} \rho\left(x_{0}\right)\right)^{d / p} \inf _{y \in Q} M_{p} f(y) .
\end{aligned}
$$

Choosing $N$ large enough, we get

$$
\begin{aligned}
\tilde{I}_{2}(x) \lesssim & \sum_{j=1}^{\infty}\left(1+2^{j}\right)^{-N} 2^{j} \rho\left(x_{0}\right) \frac{1}{\left|B\left(x_{0}, 2^{j} \rho\left(x_{0}\right)\right)\right|}\left(j 2^{j \theta^{\prime}}[b]_{\theta}\right)^{m}\left(2^{j} \rho\left(x_{0}\right)\right)^{d / p} \\
& \times \inf _{y \in Q} M_{p} f(y)\left(2^{j} \rho\left(x_{0}\right)\right)^{d-2-d / s^{\prime}}\left(2^{j}\right)^{2-d / s} \\
\lesssim & \sum_{j=1}^{\infty} j^{m} 2^{j\left(-N+m \theta^{\prime}-d+1+d / p\right)}\left([b]_{\theta}\right)^{m} \inf _{y \in Q} M_{p} f(y) \\
\lesssim & \left([b]_{\theta}\right)^{m} \inf _{y \in Q} M_{p} f(y) .
\end{aligned}
$$

Therefore, this completes the proof.

Remark 1 It is easy to check that if the critical ball $Q$ is replaced by $2 Q$, the last lemma also holds. 
Lemma 13 Let $V \in R H_{\frac{d}{2}}$ and $b \in B M O_{\theta}(\rho)$. Then, for any $s>p_{0}^{\prime}$ and $\gamma \geq 1$, there exists a constant $C>0$ such that

$$
\int_{(2 B)^{c}}|\tilde{\mathcal{K}}(x, z)-\tilde{\mathcal{K}}(y, z)|\left|b(z)-b_{B}\right|^{m}|f(z)| d z \leq C[b]_{\theta}^{m} \inf _{u \in B} M_{s} f(u)
$$

for all $f$ and $x, y \in B=B\left(x_{0}, r\right)$ with $r<\gamma \rho\left(x_{0}\right)$. Additionally, if $q_{0}>d$, the above estimate also holds for $\mathcal{K}$ instead of $\tilde{\mathcal{K}}$.

Because the proof of this lemma is very similar to that of Lemma 6 in [8], we omit the details.

Proofs of Theorem 1 and Theorem 2 We will prove Theorem 1 via the mathematical induction and Theorem 2 follows by duality. When $m=1$, we conclude that Theorem 1 is valid by Theorem 1 in [8]. Suppose that the $L^{p}$ boundedness of $\tilde{\mathcal{R}}_{b}^{\alpha} f$ holds when $\alpha=2,3, \ldots, m-1$, where $p_{0}^{\prime}<p<\infty$. In what follows, we will prove that it is valid for $k=m$.

We start with a function $f \in L^{p}\left(\mathbb{R}^{d}\right)$ for $p_{0}^{\prime}<s<\infty$, and we notice that due to Lemma 12 we have $\tilde{\mathcal{R}}_{b}^{m} f \in L_{\text {loc }}^{1}\left(\mathbb{R}^{d}\right)$.

By using Lemma 11, Lemma 12 with $p_{0}^{\prime}<p<s$ and Remark 1, we have

$$
\begin{aligned}
\left\|\tilde{\mathcal{R}}_{b}^{m} f\right\|_{L^{q}}^{q} \leq & \int_{\mathbb{R}^{d}}\left|M_{\rho, \beta}\left(\tilde{\mathcal{R}}_{b}^{m} f\right)(x)\right|^{q} d x \\
\lesssim & \int_{\mathbb{R}^{d}}\left|M_{\rho, \gamma}^{\#}\left(\tilde{\mathcal{R}}_{b}^{m} f\right)(x)\right|^{q} d x+\sum_{k}\left|Q_{k}\right|\left(\frac{1}{\left|Q_{k}\right|} \int_{2 Q_{k}}\left|\tilde{\mathcal{R}}_{b}^{m} f(x)\right| d x\right)^{q} \\
\lesssim & \int_{\mathbb{R}^{d}}\left|M_{\rho, \gamma}^{\#}\left(\tilde{\mathcal{R}}_{b}^{m} f\right)(x)\right|^{q} d x+\left(\sum_{\alpha=1}^{m}[b]_{\theta}^{\alpha}\right)^{q}\left[\sum_{k}\left(\int_{2 Q_{k}}\left|M_{p} f(x)\right| d x\right)^{q}\right. \\
& \left.+\sum_{k}\left(\int_{2 Q_{k}}\left|M_{p}\left(\tilde{\mathcal{R}}_{b}^{\alpha} f\right)(x)\right| d x\right)^{q}\right] \\
\lesssim & \int_{\mathbb{R}^{d}}\left|M_{\rho, \gamma}^{\#}\left(\tilde{\mathcal{R}}_{b}^{m} f\right)(x)\right|^{q} d x+\left(\sum_{\alpha=1}^{m}[b]_{\theta}^{\alpha}\right)^{q}\left(\|f\|_{L^{q}}^{q}+\left\|\tilde{\mathcal{R}}_{b}^{\alpha} f\right\|_{L^{q}}^{q}\right) \\
\lesssim & \int_{\mathbb{R}^{d}}\left|M_{\rho, \gamma}^{\#}\left(\tilde{\mathcal{R}}_{b}^{m} f\right)(x)\right|^{q} d x+\left(\sum_{\alpha=1}^{m}[b]_{\theta}^{\alpha}\right)^{q}\|f\|_{L^{q}}^{q}
\end{aligned}
$$

where we use the finite overlapping property given by Lemma 10, the assumption on $\tilde{\mathcal{R}}_{b}^{\alpha}$ and the boundedness of $M_{p}$ in $L^{q}\left(\mathbb{R}^{d}\right)$ for $p<q$.

Next, we consider the term $\int_{\mathbb{R}^{d}}\left|M_{\rho, \gamma}^{\#}\left(\tilde{\mathcal{R}}_{b}^{m} f\right)(x)\right|^{q} d x$. Our goal is to find a pointwise estimate of $M_{\rho, \gamma}^{\#}\left(\tilde{\mathcal{R}}_{b}^{m} f\right)(x)$. Let $x \in \mathbb{R}^{d}$ and $B=\left(x_{0}, r\right)$ with $r<\gamma \rho\left(x_{0}\right)$ such that $x \in B$. If $f=f_{1}+f_{2}$ with $f_{1}=f \chi_{2 Q}$, then we write

$$
\tilde{\mathcal{R}}_{b}^{m} f(x)=\sum_{\alpha=0}^{m-1} C_{\alpha, m}(b(x)-\lambda)^{m-\alpha} \tilde{\mathcal{R}}_{b}^{\alpha} f(x)+\tilde{\mathcal{R}}\left((b-\lambda)^{m} f\right)(x) .
$$

Therefore, we need to control the mean oscillation on $B$ of each term that we call $\mathcal{O}_{1}, \mathcal{O}_{2}$. 
Let $p>p_{0}^{\prime}$, by using the Hölder inequality and Lemma 6 , we obtain

$$
\begin{aligned}
\mathcal{O}_{1} & \lesssim \sum_{\alpha=0}^{m-1} \frac{1}{|B|} \int_{B}\left|(b(x)-\lambda)^{m-\alpha} \mathcal{R}_{b}^{\alpha} f(x)\right| d x \\
& \lesssim \sum_{\alpha=0}^{m-1}\left(\frac{1}{|B|} \int_{B}\left|\left(b(x)-b_{B}\right)\right|^{(m-\alpha) p^{\prime}} d x\right)^{1 / p^{\prime}}\left(\frac{1}{|B|} \int_{B}\left|\mathcal{R}_{b}^{\alpha} f(x)\right|^{p} d x\right)^{1 / p} \\
& \lesssim \sum_{\alpha=0}^{m-1}\left([b]_{\theta}\right)^{m-\alpha} M_{p}\left(\mathcal{R}_{b}^{\alpha} f\right)(x),
\end{aligned}
$$

since $r<\gamma \rho\left(x_{0}\right)$.

As for $\mathcal{O}_{2}$, let $1<\tilde{p}<p$. We split again $f=f_{1}+f_{2}$. Choose $p_{0}^{\prime}<\tilde{p}<p$ and denote $\left(1 / \tilde{p}^{\prime}\right)+$ $(1 / \tilde{p})=1$ and $v=\frac{\tilde{p} p}{p-\tilde{p}}$. Using the boundedness of $\tilde{\mathcal{R}}$ on $L^{\tilde{p}}\left(\mathbb{R}^{d}\right)$ and the Hölder inequality, we then get

$$
\begin{aligned}
\mathcal{O}_{2,1} & \lesssim \frac{1}{|B|} \int_{B}\left|\tilde{\mathcal{R}}_{b}\left(\left(b-b_{B}\right)^{m} f_{1}\right)(x)\right| d x \\
& \lesssim\left(\frac{1}{|B|} \int_{B}\left|\tilde{\mathcal{R}}_{b}\left(\left(b-b_{B}\right)^{m} f_{1}\right)(x)\right|^{\tilde{p}} d x\right)^{1 / \tilde{p}} \\
& \lesssim\left(\frac{1}{|B|} \int_{B} \tilde{\mathcal{R}}_{b}\left(\left|\left(b-b_{B}\right)^{m} f_{1}(x)\right|^{\tilde{s}}\right) d x\right)^{1 / \tilde{s}} \\
& \lesssim\left(\frac{1}{|B|} \int_{2 B}\left|\left(b(x)-b_{B}\right)\right|^{m v} d x\right)^{1 / v}\left(\frac{1}{|B|} \int_{2 B}|f(x)|^{p} d x\right)^{1 / p} \\
& \lesssim\left([b]_{\theta}\right)^{m} M_{p}(f)(x) .
\end{aligned}
$$

For $\mathcal{O}_{2,2}$, by Lemma 13 , we obtain

$$
\begin{aligned}
\mathcal{O}_{2,2} & \lesssim \frac{1}{|B|^{2}} \iint_{B}\left|\tilde{\mathcal{R}}_{b}\left(\left(b-b_{B}\right)^{m} f_{2}\right)(u)-\tilde{\mathcal{R}}_{b}\left(\left(b-b_{B}\right)^{m} f_{2}\right)(y)\right| d u d y \\
& \lesssim\left([b]_{\theta}\right)^{m} M_{p} f(x),
\end{aligned}
$$

since the integral is clearly bounded by the left-hand side of (13).

Therefore, we have proved that

$$
\left|M_{\rho, \gamma}^{\#}\left(\tilde{\mathcal{R}}_{b}^{m} f\right)(x)\right| \leq C \sum_{\alpha=0}^{m-1}\left([b]_{\theta}\right)^{m-\alpha}\left[M_{p}(f)(x)+M_{p}\left(\mathcal{R}_{b}^{\alpha} f\right)(x)\right] .
$$

By the assumption on $\mathcal{R}_{b}^{\alpha}$ and the $L^{p}$ boundedness of $M_{p}$, we obtain the desired result.

Proof of Theorem 3 We will prove Theorem 3 using the mathematical induction. When $m=1$, we conclude that Theorem 3 is valid by Theorem 5 in [17]. Suppose that Theorem 3 holds when $\alpha=2,3, \ldots, m-1$. In what follows, we will prove that it is valid for $k=m$.

Similarly, we only consider the case of $\frac{d}{2}<s<d$. For $f \in H_{L}^{1}\left(\mathbb{R}^{d}\right)$, we can write $f=$ $\sum_{j=-\infty}^{\infty} \lambda_{j} a_{j}$, where each $a_{j}$ is a $(1, q)_{\rho}$ atom and $\sum_{j=-\infty}^{\infty}\left|\lambda_{j}\right| \leq 2\|f\|_{H_{L}^{1}}$. Suppose that $\sup a_{j} \subseteq$ 
$B_{j}=B\left(x_{j}, r_{j}\right)$ with $r_{j}<\rho\left(x_{j}\right)$. Write

$$
\begin{aligned}
\mathcal{R}_{b}^{m} f(x)= & \lambda_{j} \mathcal{R}_{b}^{m}\left(\sum_{j=-\infty}^{\infty} a_{j}\right)(x) \\
= & \lambda_{j} \sum_{\alpha=0}^{m-1} C_{\alpha, m}\left(b(x)-b_{B_{j}}\right)^{m-\alpha} \mathcal{R}_{b}^{\alpha}\left(\sum_{j=-\infty}^{\infty} a_{j}\right)(x)+\mathcal{R}\left(\lambda_{j}\left(b-b_{B_{j}}\right)^{m} \sum_{j=-\infty}^{\infty} a_{j}\right)(x) \\
= & \sum_{\alpha=0}^{m-1} \sum_{j=-\infty}^{\infty} C_{\alpha, m} \lambda_{j}\left(b(x)-b_{B_{j}}\right)^{m-\alpha} \mathcal{R}_{b}^{\alpha} a_{j} \chi_{8 B_{j}}(x) \\
& +\sum_{\alpha=0}^{m-1} \sum_{j: r_{j} \geq \frac{\rho\left(x_{j}\right)}{4}} C_{\alpha, m} \lambda_{j}\left(b(x)-b_{B_{j}}\right)^{m-\alpha} \mathcal{R}_{b}^{\alpha} a_{j} \chi_{\left(8 B_{j}\right)^{c}}(x) \\
& +\sum_{\alpha=0}^{m-1} \sum_{j: r_{j}<\frac{\rho\left(x_{j}\right)}{4}} C_{\alpha, m} \lambda_{j}\left(b(x)-b_{B_{j}}\right)^{m-\alpha} \mathcal{R}_{b}^{\alpha} a_{j} \chi_{\left(8 B_{j}\right)^{c}}(x) \\
& +\mathcal{R}\left(\sum_{j=-\infty}^{\infty} \lambda_{j}\left(b-b_{B_{j}}\right)^{m} a_{j}\right)(x) \\
= & A_{1}(x)+A_{2}(x)+A_{3}(x)+A_{4}(x) .
\end{aligned}
$$

For the term $A_{1}(x)$, by Lemma 6 and Theorem 1, we obtain

$$
\begin{aligned}
& \left\|\left(b(x)-b_{B_{j}}\right)^{m-\alpha} \mathcal{R}_{b}^{\alpha} a_{j} \chi_{8 B_{j}}(x)\right\|_{L^{1}\left(\mathbb{R}^{d}\right)} \\
& \quad \lesssim\left(\int_{8 B_{j}}\left|\left(b(x)-b_{B_{j}}\right)^{m-\alpha}\right|^{q^{\prime}} d x\right)^{1 / q^{\prime}}\left\|\mathcal{R}_{b}^{\alpha} a_{j}\right\|_{L^{q}\left(\mathbb{R}^{d}\right)} \\
& \quad \lesssim\left(\int_{8 B_{j}}\left|\left(b(x)-b_{B_{j}}\right)^{m-\alpha}\right|^{q^{\prime}} d x\right)^{1 / q^{\prime}}\left\|a_{j}\right\|_{L^{q}\left(\mathbb{R}^{d}\right)} \\
& \lesssim\left(\frac{1}{\left|B_{j}\right|} \int_{8 B_{j}}\left|\left(b(x)-b_{B_{j}}\right)^{m-\alpha}\right|^{q^{\prime}} d x\right)^{1 / q^{\prime}} \\
& \quad \lesssim[b]_{\theta}^{m-\alpha},
\end{aligned}
$$

since $r_{j}<\rho\left(x_{j}\right)$.

Secondly, we consider the term $A_{2}(x)$. It is easy to see that $\left|x-x_{j}\right| \sim|x-y|$ and

$$
\left(1+\frac{|x-y|}{\rho(x)}\right) \geq C\left(1+\frac{\left|x-x_{j}\right|}{\rho(x)}\right) \geq C c\left(1+\frac{\left|x-x_{j}\right|}{\rho\left(x_{j}\right)}\right)^{\frac{1}{l_{0}+1}}
$$

Note that $\rho\left(x_{j}\right)>r_{j} \geq \frac{\rho\left(x_{j}\right)}{4}$. By the Hölder inequality and inequality (11), we obtain, for some $t>1$,

$$
\begin{aligned}
& \int_{2^{k} r_{j} \leq\left|x-x_{j}\right|<2^{k+1} r_{j}}\left(b(x)-b_{B_{j}}\right)^{m-\alpha}|\mathcal{K}(x, y)| d x \\
& \quad \leq\left(\int_{2^{k} r_{j} \leq\left|x-x_{j}\right|<2^{k+1} r_{j}}\left(b(x)-b_{B_{j}}\right)^{t^{\prime}(m-\alpha)} d x\right)^{\frac{1^{\prime}}{t}}\left(\int_{2^{k} r_{j} \leq\left|x-x_{j}\right|<2^{k+1} r_{j}}|\mathcal{K}(x, y)|^{t} d x\right)^{\frac{1}{t}}
\end{aligned}
$$


Wang and Lu Journal of Inequalities and Applications 2014, 2014:466

Page 13 of 16

http://www.journalofinequalitiesandapplications.com/content/2014/1/466

$$
\begin{aligned}
& \lesssim[b]_{\theta}^{m-\alpha}\left(\int_{2^{k} r_{j} \leq\left|x-x_{j}\right|<2^{k+1} r_{j}}|\mathcal{K}(x, y)|^{t} d x\right)^{\frac{1}{t}}\left(2^{k+1} r_{j}\right)^{\frac{d}{t^{\prime}}} \\
& \lesssim[b]_{\theta}^{m-\alpha}\left(1+\frac{2^{k} r_{j}}{\rho\left(x_{j}\right)}\right)^{-\frac{N}{l_{0}+1}} \frac{\left(2^{k+1} r_{j}\right)^{\frac{d}{t^{\prime}}}}{\left(2^{k} r_{j}\right)^{d-1}} \\
& \times\left(\int_{2^{k} r_{j} \leq\left|x-x_{j}\right|<2^{k+1} r_{j}}\left|\left(\int_{B\left(x_{j}, 2^{k+3} r_{j}\right)} \frac{V(z)}{\left|z-x_{j}\right|^{d-1}} d z\right)\right|^{t} d x\right)^{\frac{1}{t}} \\
& +[b]_{\theta}^{m-\alpha}\left(1+\frac{2^{k} r_{j}}{\rho\left(x_{j}\right)}\right)^{-\frac{N}{l_{0}+1}}\left(2^{k+1} r_{j}\right)^{\frac{d}{t^{\prime}}}\left(\int_{2^{k} r_{j} \leq\left|x-x_{j}\right|<2^{k+1} r_{j}} \frac{1}{\left|x-x_{j}\right|^{(d+\delta) t}} d x\right)^{\frac{1}{t}} \\
& \lesssim[b]_{\theta}^{m-\alpha}\left(1+\frac{2^{k} r_{j}}{\rho\left(x_{j}\right)}\right)^{-\frac{N}{l_{0}+1}} \frac{\left(2^{k+1} r_{j}\right)^{\frac{d}{t^{\prime}}}}{\left(2^{k} r_{j}\right)^{d-1}}\left(\left(\int_{B\left(x_{j}, 2^{k+3} r_{j}\right)} V^{q}(z) d z\right)^{\frac{1}{q}}+1\right) \\
& \lesssim[b]_{\theta}^{m-\alpha}\left(1+\frac{2^{k} r_{j}}{\rho\left(x_{j}\right)}\right)^{-\frac{N}{l_{0}+1}} \frac{\left(2^{k+1} r_{j}\right)^{\frac{d}{t^{\prime}}}}{\left(2^{k} r_{j}\right)^{d-1}}\left(\left(2^{k+3} r_{j}\right)^{d}\left(\int_{B\left(x_{j}, 2^{k+3} r_{j}\right)} V(z) d z\right)\left(2^{k+3} r_{j}\right)^{\frac{d}{q}}+1\right) \\
& \lesssim[b]_{\theta}^{m-\alpha}\left(1+2^{k} r_{j} \rho\left(x_{j}\right)\right)^{-\frac{N}{l_{0}+1}+l_{1}}\left(\frac{\left(2^{k+1} r_{j}\right)^{\frac{d}{t^{\prime}}}}{\left(2^{k} r_{j}\right)^{d-1}}\left(2^{k} r_{j}\right)^{-2+\frac{d}{q}}+1\right) \\
& \lesssim[b]_{\theta}^{m-\alpha}\left(1+\frac{2^{k} r_{j}}{\rho\left(x_{j}\right)}\right)^{-\frac{N}{l_{0}+1}+l_{1}}
\end{aligned}
$$

where $\frac{1}{t}=\frac{1}{q}-\frac{1}{d}$. Via the above estimate, we have

$$
\begin{aligned}
& \left\|\left(b(x)-b_{B_{j}}\right)^{m-\alpha} \mathcal{R}_{b}^{\alpha} a_{j} \chi_{\left(8 B_{j}\right)}(x)\right\|_{L^{1}\left(\mathbb{R}^{d}\right)} \\
& \quad \leq \int_{\left|x-x_{j}\right| \geq 8 B_{j}}\left(b(x)-b_{B_{j}}\right)^{m-\alpha}\left|\int_{B_{j}} K(x, y) a_{j}(y) d y\right| d x \\
& \quad \leq \int_{8 B_{j}}\left|a_{j}(y)\right| \sum_{k=1}^{\infty} \int_{2^{k} r_{j} \leq\left|x-x_{j}\right|<2^{k+1} r_{j}}\left(b(x)-b_{B_{j}}\right)^{m-\alpha}|K(x, y)| d x d y \\
& \quad \leq \int_{8 B_{j}}\left|a_{j}(y)\right| \sum_{k=1}^{\infty}[b]_{\theta}^{m-\alpha}\left(1+\frac{2^{k} r_{j}}{\rho\left(x_{j}\right)}\right)^{-\frac{N}{l^{+1}}+l_{1}} d y \\
& \quad \lesssim[b]_{\theta}^{m-\alpha}
\end{aligned}
$$

if we choose $N$ large enough.

Thirdly, we consider the term $A_{3}(x)$. Via the Holder inequality and (12), we get, for some $t>1$,

$$
\begin{aligned}
& \int_{2^{k} r_{j} \leq\left|x-x_{j}\right|<2^{k+1} r_{j}}\left(b(x)-b_{B_{j}}\right)^{m-\alpha}\left|\mathcal{K}(x, y)-\mathcal{K}\left(x, x_{j}\right)\right| d x \\
& \leq\left(\int_{2^{k} r_{j} \leq\left|x-x_{j}\right|<2^{k+1} r_{j}}\left(b(x)-b_{B_{j}}\right)^{t^{\prime}(m-\alpha)} d x\right)^{\frac{1^{\prime}}{t}} \\
& \quad \times\left(\int_{2^{k} r_{j} \leq\left|x-x_{j}\right|<2^{k+1} r_{j}}\left|\mathcal{K}(x, y)-\mathcal{K}\left(x, x_{j}\right)\right|^{t} d x\right)^{\frac{1}{t}}
\end{aligned}
$$




$$
\begin{aligned}
& \lesssim[b]_{\theta}^{m-\alpha}\left(\int_{2^{k} r_{j} \leq\left|x-x_{j}\right|<2^{k+1} r_{j}}\left|\mathcal{K}(x, y)-\mathcal{K}\left(x, x_{j}\right)\right|^{t} d x\right)^{\frac{1}{t}}\left(2^{k+1} r_{j}\right)^{\frac{d}{t^{\prime}}} \\
& \lesssim[b]_{\theta}^{m-\alpha}\left(1+\frac{2^{k} r_{j}}{\rho\left(x_{j}\right)}\right)^{-\frac{N}{l_{0}+1}} \frac{\left(r_{j}\right)^{\delta}\left(2^{k+1} r_{j}\right)^{\frac{d}{t^{\prime}}}}{\left(2^{k} r_{j}\right)^{d-1+\delta}} \\
& \times\left(\int_{2^{k} r_{j} \leq\left|x-x_{j}\right|<2^{k+1} r_{j}}\left|\left(\int_{B\left(x_{j}, 2^{k+3} r_{j}\right)} \frac{V(z)}{\left|z-x_{j}\right|^{d-1}} d z\right)\right|^{t} d x\right)^{\frac{1}{t}} \\
& +[b]_{\theta}^{m-\alpha}\left(1+\frac{2^{k} r_{j}}{\rho\left(x_{j}\right)}\right)^{-\frac{N}{l_{0}+1}}\left(r_{j}\right)^{\delta}\left(2^{k+1} r_{j}\right)^{\frac{d}{t^{\prime}}} \\
& \times\left(\int_{2^{k} r_{j} \leq\left|x-x_{j}\right|<2^{k+1} r_{j}} \frac{1}{\left|x-x_{j}\right|^{(d+\delta) t}} d x\right)^{\frac{1}{t}} \\
& \lesssim[b]_{\theta}^{m-\alpha} \frac{1}{\left\{1+\frac{2^{k} r_{j}}{\rho\left(x_{j}\right)}\right\}^{\frac{N}{l^{+1}}}}\left\{\frac{r_{j}^{\delta}\left(2^{k+1} r_{j}\right)^{\frac{d}{t^{\prime}}}}{\left(2^{k} r_{j}\right)^{d-1+\delta}}\left(\int_{B\left(x_{j}, 2^{k+3} r_{j}\right)} V(z)^{q} d z\right)^{\frac{1}{q}}+2^{-k \delta}\right\} \\
& \lesssim[b]_{\theta}^{m-\alpha} \frac{1}{\left\{1+\frac{2^{k} r_{j}}{\rho\left(x_{j}\right)}\right\}^{\frac{N}{l_{0}+1}}} \\
& \times\left\{\frac{r_{j}^{\delta}\left(2^{k+1} r_{j}\right)^{\frac{d}{t^{\prime}}}}{\left(2^{k} r_{j}\right)^{d-1+\delta}}\left(\frac{1}{\left(2^{k+3} r_{j}\right)^{d}} \int_{B\left(x_{j}, 2^{k+3} r_{j}\right)} V(z) d z\right)\left(2^{k} r_{j}\right)^{\frac{d}{q}}+2^{-k \delta}\right\} \\
& \lesssim[b]_{\theta}^{m-\alpha} \frac{1}{\left\{1+\frac{2^{k} r_{j}}{\rho\left(x_{j}\right)}\right\}^{\frac{N}{l_{0}+1}-l_{1}}}\left(r_{j}^{\delta} \frac{\left(2^{k+1} r_{j}\right)^{\frac{d}{t^{\prime}}}}{\left(2^{k} r_{j}\right)^{d-1+\delta}}\left(2^{k} r_{j}\right)^{-2+\frac{d}{q}}+2^{-k \delta}\right) \\
& \lesssim[b]_{\theta}^{m-\alpha} \frac{1}{\left\{1+\frac{2^{k} r_{j}}{\rho\left(x_{j}\right)}\right\}^{\frac{N}{l_{0}+1}-l_{1}}} 2^{-k \delta},
\end{aligned}
$$

where $\frac{1}{t}=\frac{1}{q}-\frac{1}{d}$.

Similarly, via the above estimate and the vanishing moment of $a_{j}$, we have

$$
\begin{aligned}
& \left\|\left(b(x)-b_{B_{j}}\right)^{m-\alpha} \mathcal{R}_{b}^{\alpha} a_{j} \chi_{\left(8 B_{j}\right)^{c}}(x)\right\|_{L^{1}\left(\mathbb{R}^{d}\right)} \\
& \quad \leq \int_{\left|x-x_{j}\right| \geq 8 B_{j}}\left(b(x)-b_{B_{j}}\right)^{m-\alpha}\left|\int_{B_{j}}\left[\mathcal{K}(x, y)-\mathcal{K}\left(x, x_{j}\right)\right] a_{j}(y) d y\right| d x \\
& \quad \leq \int_{8 B_{j}}\left|a_{j}(y)\right| \sum_{k=1}^{\infty} \int_{2^{k} r_{j} \leq\left|x-x_{j}\right|<2^{k+1} r_{j}}\left(b(x)-b_{B_{j}}\right)^{m-\alpha}\left|\left[\mathcal{K}(x, y)-\mathcal{K}\left(x, x_{j}\right)\right]\right| d x d y \\
& \quad \lesssim[b]_{\theta}^{m-\alpha} \int_{8 B_{j}}\left|a_{j}(y) d y\right| \sum_{k=1}^{\infty} \frac{1}{\left\{1+\frac{2^{k} r_{j}}{\rho\left(x_{j}\right)}\right\}^{\frac{N}{l_{0}+1}-l_{1}}} 2^{-k \delta} \\
& \quad \lesssim[b]_{\theta}^{m-\alpha} .
\end{aligned}
$$

Thus, we have

$$
\left|\left\{x \in \mathbb{R}^{d}:\left|A_{i}(x)\right|>\frac{\lambda}{4}\right\}\right| \leq \frac{C}{\lambda}\left\|A_{i}(x)\right\|_{L_{1}} \lesssim \frac{\sum_{\alpha=1}^{m}[b]_{\theta}^{\alpha}}{\lambda} \sum_{j=-\infty}^{\infty}\left|\lambda_{j}\right|, \quad i=1,2,3
$$


Moreover, note that

$$
\begin{aligned}
\left\|\left(b-b_{B_{j}}\right)^{m} a_{j}\right\|_{L^{1}} & \leq\left(\int_{B_{j}}\left(b(x)-b_{B_{j}}\right)^{m q^{\prime}} d y\right)^{1 / q^{\prime}}\left\|a_{j}\right\|_{L^{q}} \\
& \leq\left(\frac{1}{\left|B_{j}\right|} \int_{B_{j}}\left(b(x)-b_{B_{j}}\right)^{m q^{\prime}} d y\right)^{1 / q^{\prime}} \\
& \leq[b]_{\theta}^{m}\left(1+\frac{r_{j}}{\rho\left(x_{j}\right)}\right)^{\theta^{\prime} m} \\
& \lesssim[b]_{\theta}^{m},
\end{aligned}
$$

where $r_{j}<\rho\left(x_{j}\right)$.

By the weak $(1,1)$ boundedness of $\mathcal{R}$, we get

$$
\begin{aligned}
\left|\left\{x \in \mathbb{R}^{d}:\left|A_{4}(x)\right|>\frac{\lambda}{4}\right\}\right| & \lesssim \frac{1}{\lambda}\left\|\sum_{j=-\infty}^{\infty} \lambda_{j}\left(b-b\left(x_{j}\right)\right) a_{j}\right\|_{L_{1}} \\
& \lesssim \frac{[b]_{\theta}^{m}}{\lambda} \sum_{j=-\infty}^{\infty}\left|\lambda_{j}\right| .
\end{aligned}
$$

Therefore,

$$
\begin{aligned}
\left|\left\{x \in \mathbb{R}^{d}:\left|\mathcal{R}_{b}^{m} f(x)\right|>\frac{\lambda}{4}\right\}\right| & \lesssim \sum_{i=1}^{4}\left|\left\{x \in \mathbb{R}^{d}:\left|A_{i}\right|>\frac{\lambda}{4}\right\}\right| \\
& \lesssim \frac{\sum_{\alpha=1}^{m}[b]_{\theta}^{\alpha}}{\lambda} \sum_{j=-\infty}^{\infty}\left|\lambda_{j}\right| \\
& \lesssim \frac{\sum_{\alpha=1}^{m}[b]_{\theta}^{\alpha}}{\lambda}\|f\|_{H_{L}^{1}}
\end{aligned}
$$

This completes the proof of Theorem 3.

\section{Competing interests}

The authors declare that they have no competing interests.

\section{Authors' contributions}

All authors contributed equally to the writing of this paper. All authors read and approved the final manuscript.

\section{Acknowledgements}

The second author would like to thank Prof. Jie Xiao and the Department of Mathematics and Statistics of Memorial University of Newfoundland for their hospitality. This work is supported by the National Natural Science Foundation of China (Nos. 10901018, 11471018), the Fundamental Research Funds for the Central Universities (No. FRF-TP-14-005C1), Program for New Century Excellent Talents in University and the Beijing Natural Science Foundation under Grant (No. 1142005)

\section{Received: 8 September 2014 Accepted: 12 November 2014 Published: 26 Nov 2014}

\section{References}

1. Segovia, C, Torrea, JL: Higher order commutators for vector-valued Calderón-Zygmund operators. Trans. Am. Math. Soc. 336(2), 537-556 (1993)

2. Ding, Y, Lu, SZ: Higher order commutators for a class of rough operators. Ark. Mat. 37, 33-44 (1999)

3. Ding, Y, Lu, SZ, Zhang, P: Continuity of higher order commutators on certain Hardy spaces. Acta Math. Sin. Engl. Ser. 18(2), 391-404 (2002) 
4. Ding, $Y, L u, S Z$ : Weighted $L^{p}$ boundedness for higher order commutators of oscillatory singular integrals. Tohoku Math. J. 48, 437-449 (1996)

5. Pérez, C: Endpoint estimates for commutators of singular integral operators. J. Funct. Anal. 128(1), 163-185 (1995)

6. Liu, HP, Tang, L: Compactness for higher order commutators of oscillatory singular integral operators. Int. J. Math. 20(9), 1137-1146 (2009)

7. Guo, Z, Li, P, Peng, L: $L^{p}$ Boundedness of commutators of Riesz transforms associated to Schrödinger operator. J. Math. Anal. Appl. 341(1), 421-432 (2008)

8. Bongioanni, B, Harboure, E, Salinas, O: Commutators of Riesz transforms related to Schrödinger operators. J. Fourier Anal. Appl. 17(1), 115-134 (2011)

9. Bongioanni, B, Harboure, E, Salinas, O: Weighted inequalities for commutators of Schrödinger Riesz transforms. J. Math. Anal. Appl. 392, 6-22 (2012)

10. Li, PT, Peng, LZ: Endpoint estimates for commutators of Riesz transforms associated with Schrödinger operators. Bull. Aust. Math. Soc. 82(3), 367-389 (2010)

11. Li, PT, Peng, LZ: Compact commutators of Riesz transforms associated to Schrödinger operator. Pure Appl. Math. Q. 8(3), 713-739 (2012)

12. $L i, P T$, Peng, $L Z: L^{D}$ Boundedness of commutator operator associated with Schrödinger operators on Heisenberg group. Acta Math. Sci. 32(2), 568-578 (2012)

13. Liu, Y, Wang, L, Dong, J: Commutators of higher order Riesz transform associated with Schrödinger operators. J. Funct. Spaces Appl. 2013, Article ID 842375 (2013)

14. Liu, Y: Commutators of $B M O$ functions and degenerate Schrödinger operators with certain nonnegative potentials. Monatshefte Math. 165, 41-56 (2012)

15. Liu, Y, Huang, JZ, Dong, JF: Commutators of Calderón-Zygmund operators related to admissible functions on spaces of homogeneous type and applications to Schrödinger operators. Sci. China Math. 56(9), 1895-1913 (2013)

16. Liu, Y, Sheng, JL: Some estimates for commutators of Riesz transforms associated with Schrödinger operators. J. Math Anal. Appl. 419, 298-328 (2014)

17. Liu, Y, Sheng, JL, Wang, L: Weighted endpoint estimates for commutators of Riesz transforms associated with Schrödinger operators. Abstr. Appl. Anal. 2013, Article ID 281562 (2013)

18. Yu, WX, Wang, XY: Weighted sharp function inequalities and boundedness for commutator of Riesz transforms of Schrödinger operator. Integral Transforms Spec. Funct. 25(10), 765-776 (2014)

19. Dziubański, J, Zienkiewicz, J: Hardy spaces $H^{1}$ associated to Schrödinger operators with potential satisfying reverse Hölder inequality. Rev. Mat. Iberoam. 15(2), 279-296 (1999)

20. Shen, ZW: $L^{P}$ Estimates for Schrödinger operators with certain potentials. Ann. Inst. Fourier (Grenoble) 45, 513-546 (1995)

21. Garcia-Cuerva, J, Harboure, E, Segovia, C, et al.: Weighted norm inequalities for commutators of strongly singular integrals. Indiana Univ. Math. J. 40(4), 1397-1420 (1991)

10.1186/1029-242X-2014-466

Cite this article as: Wang and Liu: Higher order commutators of Riesz transforms related to Schrödinger operators. Journal of Inequalities and Applications 2014, 2014:466

\section{Submit your manuscript to a SpringerOpen ${ }^{\circ}$ journal and benefit from:}

- Convenient online submission

Rigorous peer review

- Immediate publication on acceptance

- Open access: articles freely available online

- High visibility within the field

- Retaining the copyright to your article 